\title{
Towards bipolar tin monoxide: Revealing unexplored dopants
}

\author{
Migle Graužinyte, ${ }^{*}$ Stefan Goedecker, and José A. Flores-Livas ${ }^{\dagger}$ \\ Department of Physics, Universität Basel, Klingelbergstr. 82, 4056 Basel, Switzerland
}

(Received 21 June 2018; revised manuscript received 28 August 2018; published 30 October 2018)

\begin{abstract}
The advancement of transparent electronics, one of the most anticipated technological developments for the future, is currently inhibited by a shortage of high-performance $p$-type semiconductors. Recent demonstration of tin monoxide as a successful transparent $p$-type thin-film transistor and the discovery of its potential for ambipolar doping, suggests that tin monoxide—an environmentally friendly earth-abundant material—could offer a solution to this challenge. With the aim of enhancing the electronic properties, an extensive search for useful dopant elements was performed. Substitutional doping with the family of alkali metals was identified as a successful route to increase the concentration of acceptors in $\mathrm{SnO}$ and over ten shallow donors, which, to the best of our knowledge, have not been previously contemplated, were discovered. This work presents a detailed analysis of the most promising $n$-/ $p$-type dopants-offering new insights into the design of an ambipolar $\mathrm{SnO}$. If synthesized successfully, such a doped ambipolar oxide could open new avenues for many transparent technologies.
\end{abstract}

DOI: 10.1103/PhysRevMaterials.2.104604

\section{INTRODUCTION}

Tin monoxide ( $\mathrm{SnO})$, the less coveted brother of the well established $n$-type transparent conductor tin dioxide $\left(\mathrm{SnO}_{2}\right)$, has recently recaptured the attention of the scientific community due to its $p$-type properties and the promise of ambipolar doping [1-5]. The layered crystalline structure of $\mathrm{SnO}$ results in a fundamental band gap of only $0.7 \mathrm{eV}$ [2]. Yet, its indirect nature implies a much larger optical gap of $2.7 \mathrm{eV}[2,6]$ —close to the requirements for transparency in the visible spectrum. As a consequence, this oxide material shows potential for diverse applications, such as novel optoelectronic devices, thin-film transistors (TFT), thermoelectric devices, and transparent flexible circuits [7-11]. Moreover, a methodology for harvesting single monolayers of optically transparent semiconducting $\mathrm{SnO}$ from an interfacial oxide layer of liquid tin has recently been demonstrated and used to fabricate a $p$-type field-effect transistor [12].

Oxide semiconductors offer advantages over amorphous silicon in use for TFTs as they are generally more uniform and can be used with established methods for large-area and lowtemperature deposition [13]. However, $p$-type channel oxide materials have proven elusive with the family of $\mathrm{Cu}_{2} \mathrm{O}$-based materials garnering most of the attention. While hybridization of $\mathrm{Cu}-d$ and $\mathrm{O}-p$ orbitals allows for $p$-type conductivity, difficulties with generating appropriate hole densities and mobilities remain [14].

The existence of ambipolar semiconductors, materials that can exhibit both $p$-type and $n$-type behavior within a single phase, suggests an alternative route to attaining $p$-type conductivity in transparent materials. Acceptor doping of a transparent conductive oxide (TCO) that exhibits native

\footnotetext{
*migle.grauzinyte@unibas.ch

†jose.flores@unibas.ch
}

$n$-type character, such as $\mathrm{SnO}_{2}$ and $\mathrm{ZnO}$, would not only result in a transparent $p$-type conductor, but would allow for the first fully transparent $p-n$ homojunction to be realized. Synthesizing such homojunctions is crucial for the functionality of the next-generation optoelectronics $[15,16]$. Unfortunately, only limited success in creating $p$-type TCOs in such a way has been achieved, with hole concentrations in the materials remaining discouragingly low $[17,18]$. In comparison, $\mathrm{SnO}$ has been successfully demonstrated as a transparent $p$-type TFT [10] and the possibility of doping $\mathrm{SnO} n$-type with antimony has been shown as early as 2010 [4]. Furthermore, $n$-type doping of $\mathrm{SnO}$ is theoretically predicted to be beneficial for thermoelectric applications [9]. Despite this, the full range of possibilities for bipolar doping of tin monoxide remains, to this day, largely unexplored.

The ambipolar nature of $\mathrm{SnO}$ is explained by the ionization potential of the material (experimentally reported to lie in the range of $4.9-5.8 \mathrm{eV}[4,19])$ similar to other $p$-type semiconductors. Unlike most $\mathrm{Cu}_{2} \mathrm{O}$-based $p$-type TCOs with band gaps of over $2 \mathrm{eV}$, the fundamental band gap of $\mathrm{SnO}$ is strikingly small and, hence, allows for an electron affinity similar to that of $n$-type conductors to be simultaneously attained. Furthermore, the sizable band dispersions around both the conduction band minimum (CBM) and the valence band maximum (VBM), shown in the left panel of Fig. 1, result in moderate effective masses for charge carriers of either flavor [3]. In addition, a large gap of about $4 \mathrm{eV}$ between the lowest and the second lowest unoccupied bands in $\mathrm{SnO}$ prevents strong degradation of transparency due to free-carrier absorption, when electron concentrations are increased [20]. All of the above characteristics hint towards an excellent ambipolar TCO candidate.

Previous studies using Kohn-Sham density-functional theory unanimously agree that cation vacancies, $\mathrm{V}_{\mathrm{Sn}}$, act as shallow acceptors in SnO [3,5,6]. Although, most authors claim 

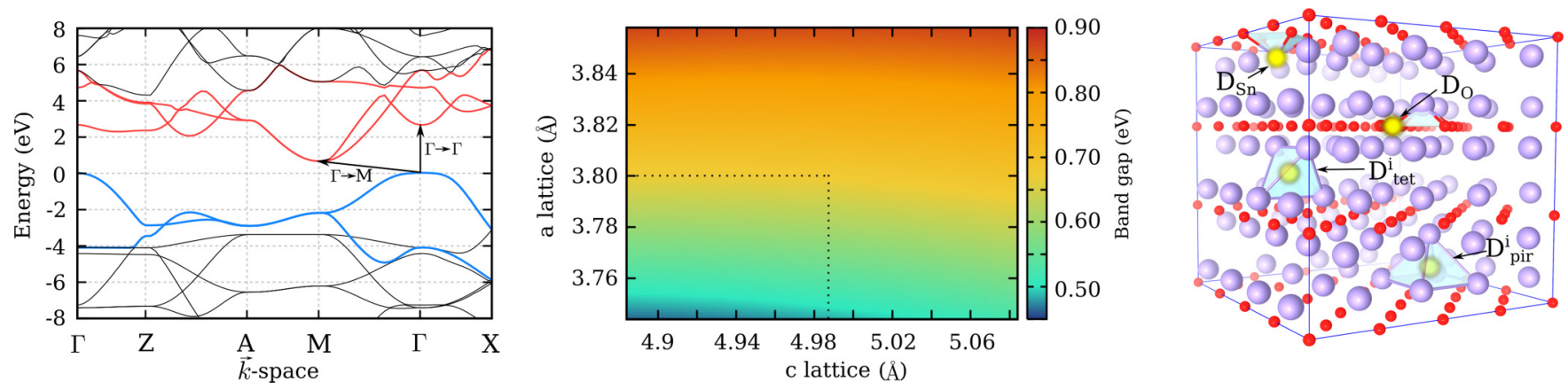

FIG. 1. (Left) The electronic band structure of SnO (HSE06). Arrows show the indirect $(\Gamma \rightarrow M)$ and the direct $(\Gamma \rightarrow \Gamma)$ band gap. (Middle) The dependence of the indirect electronic energy gap (HSE06) on the lattice parameters of the litharge SnO. (Right) Nomenclature for the different doping sites in crystalline SnO. Oxygen atoms are represented in red, tin atoms in light-purple, yellow spheres highlight the defect sites.

this to be the source of the unintentional $p$-type conductivity, Varley et al. [5] point out that the shallow behavior of tin vacancies is an insufficient criteria for copious charge carrier generation, as the high formation energy would limit the equilibrium concentration of this defect. The authors further hint that unintentional hydrogen incorporation may be key to understanding the electronic properties of SnO. Hydrogen impurities can form complexes with tin vacancies, lowering the formation energy of these defects, while maintaining their acceptor nature. The same research group was the only one to date to provide theoretical insights into external doping of tin monoxide with the aim of improving electrical properties, though only $\mathrm{H}$ and $\mathrm{Sb}$ were considered [5].

Experimentally, the story is less transparent. A link between cation deficiency and an increase in conductivity, that would support the theoretical studies above, has been reported [21]. On the other hand, cation excess resulting in Sn-metal cluster formation inside the $\mathrm{SnO}$ thin films was also demonstrated to lead to significantly improved $p$-type properties $[7,10]$. Both yttrium and antimony doping have been reported to enhance the $p$-type conductivity of $\mathrm{SnO}$ by Guo et al. [21], while Hosono et al. [4] established the ambipolar nature of tin monoxide by $n$-type antimony doping. Such inconsistencies provide a glimpse into the challenging nature of high-quality doped $\mathrm{SnO}$ thin-film production. In contrast, the advances in theoretical methodologies [22] and computational power in the past decades has made it feasible to study doped materials in silico. The information gained from such an exploratory work could reduce significantly the experimental efforts needed to produce $n-/ p$-type $\mathrm{SnO}$ films, by guiding the material synthesis towards the subspace of elements with the most enticing properties.

Notwithstanding, computational modeling of tin monoxide posses its own challenges. Standard exchange-correlation functionals, such as the generalized gradient approximation (GGA), are known to underestimate the band gaps of semiconductors. Yet, obtaining an accurate band gap is crucial for calculating reliable defect formation energies. Furthermore, the layered structure of $\mathrm{SnO}$ with interlayer interactions mediated via van der Waals (vdWs) forces is not well described by standard DFT, as dispersion interactions are not present in the exchange-correlation functionals [6].
In this work, we demonstrate that the screened hybrid HSE06 exchange-correlation functional accurately captures the band gap and the intralayer structure of tin monoxide. We further prove that the fundamental band gap of tin monoxide is not strongly dependent on the interlayer distances suggesting that HSE06 establishes a suitable level of theory for calculating defect formation energies. The above knowledge is utilized to perform a thorough scan of the entire periodic table of elements in search of useful $n$ - and $p$-type dopants. The success of doping and its repercussions on the structural, electronic and optical properties of $\mathrm{SnO}$ are discussed. This search sheds light on the possibilities of enhancing the properties of tin monoxide through targeted substitutional doping, providing a useful reference for future experiments.

\section{METHODOLOGY}

\section{A. Defect calculations}

A total of 64 elements was considered for substitutional doping in the litharge $(\mathrm{P} 4 / \mathrm{nmm})$ structure of SnO. Two different substitutional sites were investigated for each defect species: (i) a $\mathrm{Sn}$ site, further noted as $\mathrm{D}_{\mathrm{Sn}}$, where D stands for the defect species; (ii) an $\mathrm{O}$ site, further noted as $\mathrm{D}_{\mathrm{O}}$. Two additional interstitial sites were checked for atoms identified as suitable dopants in the substitutional sites, namely, (iii) a site in the middle of a pyramid formed by five $\mathrm{Sn}$ atoms, further noted as $\mathrm{D}_{\text {pir }}^{\mathrm{i}}$; and (iv) a site in the middle of a tetrahedron formed by four $\mathrm{Sn}$ atoms, further noted as $\mathrm{D}_{\text {tet }}^{\mathrm{i}}$. The four defect sites considered throughout the work are shown in the right panel of Fig. 1.

All formation energies were first evaluated with PBE level of theory and if either acceptor or donor behavior was identified, a subsequent hybrid functional calculation cycle was performed to verify the result. The choice of PBE prescreening for TCO materials is justified and demonstrated elsewhere [23].

To successfully generate free carriers, an impurity element needs to be stable in a suitable charge state. $n$-type conductivity is enhanced, when the dopant favors a positive charge state, for Fermi levels $\epsilon_{F}$ in the vicinity of the conduction band. $p$-type conductivity is enhanced if the dopant favors a negative charge state, for $\epsilon_{F}$ close to the valence band. The 
formation energy of a defect $E_{D_{x}}^{F}$ as a function of charge $q$ allows the stable charge state of a specific defect $D_{x}$ to be determined. Subsequently, its suitability for doping can be evaluated. Equation (1) was used to determine the formation energies of all defects considered in this work:

$$
\begin{aligned}
E_{D_{x}}^{F}\left(q, \Delta \epsilon_{F}\right)= & E_{D_{x}}^{q}-E_{\mathrm{SnO}}-\sum_{i} n_{i}\left[\mu_{i}+\Delta \mu_{i}\right] \\
& +q\left[\epsilon_{\mathrm{V}}+\Delta \epsilon_{F}\right]+E_{\mathrm{cor}} .
\end{aligned}
$$

Here, $E_{D_{x}}^{q}$ is the energy of a supercell with an impurity atom of species $D$ incorporated on the site $x$ in a charge state $q$; $E_{\mathrm{SnO}}$ is the energy of the unaltered $\mathrm{SnO}$ structure in the same size supercell; $n_{i}$ is the amount of atoms of type $i$ that was added $\left(n_{i}>0\right)$ or removed $\left(n_{i}<0\right)$ in order to create the defect; $\mu_{i}$ is the chemical potential of species $i$ in the elemental phase, while $\Delta \mu_{i}$ imposes the boundaries on the total chemical potential of species $i$, required to avoid secondary phase formation; $\epsilon_{V}$ is the energy of the valence band maximum of the perfect $\mathrm{SnO}$ crystal; $\Delta \epsilon_{F}$ is the Fermi level referenced to $\epsilon_{V}$. The Fermi level was regarded as a free parameter throughout the main body of the work, reflecting the $n$ - or $p$-type environments in SnO. For impurity atoms deemed as useful dopants, however, the Fermi level was also evaluated self-consistently and used to obtain equilibrium defect and charge carrier concentrations. These results are summarized in Ref. [24]. Additional correction terms were collected within the $E_{\text {cor }}$ expression. These include a potential alignment term and an anisotropic electrostatic correction term for all charged defect calculations. For shallow donor/acceptor elements, an electrostatic correction was added to all charge states exhibiting shallow behavior (including the charge neutral calculations).

Chemical potentials $\Delta \mu_{\mathrm{O}}$ and $\Delta \mu_{\mathrm{Sn}}$ are bound by $\mathrm{SnO}$ stability and limited by the possibility of oxidation into $\mathrm{SnO}_{2}$. These two criteria summarized in the equations underneath require $\Delta \mu_{\mathrm{O}}$ to lie below the difference between the formation enthalpies of $\mathrm{SnO}_{2}$ and $\mathrm{SnO}$ :

$$
\begin{gathered}
\Delta \mu_{\mathrm{Sn}}+\Delta \mu_{\mathrm{O}}=\Delta H^{F}(\mathrm{SnO}) \\
\Delta \mu_{\mathrm{Sn}}+2 \Delta \mu_{\mathrm{O}} \leqslant \Delta H^{F}\left(\mathrm{SnO}_{2}\right) .
\end{gathered}
$$

Using enthalpy values calculated with the HSE06 functional, we find $\Delta \mu_{\mathrm{O}}=-2.7 \mathrm{eV}$ and $\Delta \mu_{\mathrm{Sn}}=0.2 \mathrm{eV}$. The positive (unphysical) chemical potential of $\mathrm{Sn}$ is consistent with the experimental observation, that $\mathrm{SnO}$ is metastable with respect to decomposition into $\mathrm{SnO}_{2}$ and metallic $\mathrm{Sn}$ at high temperatures [25]. As a consequence the chemical potentials were set to $\Delta \mu_{S n}=0$ and $\Delta \mu_{\mathrm{O}}=\Delta H^{F}\left(\mathrm{SnO}_{2}\right) / 2=$ $-2.6 \mathrm{eV}$, i.e., the intersection between metallic $\mathrm{Sn}$ and the stable $\mathrm{SnO}_{2}$ phase following the approach of Ref. [6]. For all other dopant species $i$ the chemical potential, $\mu_{i}$, was evaluated using the stable crystalline, elemental phase. Secondary phase formation was considered only for those elements, that were identified as successful substitutional dopants. Structural information pertaining to all the phases used in determining the chemical potentials is listed in Ref. [24].

\section{B. Thermodynamic transition levels}

Thermodynamic transition levels $\epsilon\left(q_{1} / q_{2}\right)$ between two charged states $q_{1}$ and $q_{2}$ indicate the value of $\Delta \epsilon_{F}$ at which the stable charge state of a defect changes and are directly related to the energy required to ionize the defect. Strong structural relaxations between different charge states can create kinetic barriers that help to stabilize an excited charge state above its thermodynamic transition level [26]. Thermodynamic transition levels for a defect atom $D$ on a site $x, D_{x}$, were calculated using Eq. (2):

$$
\epsilon\left(q_{1} / q_{2}\right)=\frac{E_{D_{x}}^{F}\left(q_{1}, \Delta \epsilon_{F}=0\right)-E_{D_{x}}^{F}\left(q_{2}, \Delta \epsilon_{F}=0\right)}{q_{2}-q_{1}} .
$$

\section{Computational details}

A 192-atom supercell $(4 \times 4 \times 3)$ of $\mathrm{SnO}$ was used for all defect calculations performed in the study, corresponding to a defect concentration of $\sim 0.5 \%$. The projector augmentedwave method [27,28] as implemented in the VASP [29,30] code was used to perform the calculations. The Birch-Murnaghan fit [31] to the litharge structure of $\mathrm{SnO}$ was used to obtain the unit-cell volumes of PBE [32], PBE0 [33,34], HSE06 [35,36], and DFT-D2 as parametrized by Grimme [37] exchangecorrelation functional. Primitive cell calculations were performed using a $k=6 \times 6 \times 6$ Monkhorst-Pack mesh. An energy cutoff of $700 \mathrm{eV}$ was used for the plane waves in all calculations to obtain energy convergence of $1 \mathrm{meV} /$ atom. Sn $d$-shell electrons were included as valence states in the PAW potentials. All calculations were spin polarized.

All supercell calculations were performed using a single $\Gamma$ point only. The supercell lattice parameters were fixed to those of the expanded primitive cell and the atomic positions were allowed to relax until the forces on the atoms were below $0.002 \mathrm{eV} / \AA$. All HSE06 formation energies reported through this work were calculated by compressing the PBE-relaxed defect structures to the HSE06 lattice constant. No further HSE06 atomic relaxations were performed. A test of the influence of the relaxations on a subset of defects revealed the effect of the relaxations to be negligible on the calculated thermodynamic transition energies (see Ref. [24]).

Charge corrections were evaluated using the approach proposed by Kumagai and Oba [38] as implemented in the PYCDT code [39]. Estimation of the electrostatic correction term requires the evaluation of the static dielectric constant of $\mathrm{SnO}$. Both electronic (and ionic) contributions of the dielectric constant were evaluated using the PBE functional. The values $\epsilon_{\|}=7.405(15.054)$ and $\epsilon_{\perp}=$ $6.739(5.221)$ were obtained. Only the electronic contribution including local field effects, was evaluated using the HSE06 functional, resulting in $\epsilon_{\|}=6.974$ and $\epsilon_{\perp}=6.096$. $\mathrm{PBE}$ ratios between the electronic and ionic contributions were then used to estimate the ionic contribution for HSE06, resulting in the values $\epsilon_{\|}=(14.177)$ and $\epsilon_{\perp}=$ (4.722). Our estimated average dielectric constant of 17.7 is within the range of experimentally reported relative permittivities of 15-18.8 [40,41]. We note that at such large sizes of the supercell and values of the average dielectric constant the final results are not strongly sensitive to the exact value used for the dielectric constant. A change of $\sim 23 \mathrm{meV}$ in the electrostatic 
charge correction was calculated for the singly ionized and $\sim 81 \mathrm{meV}$ for the doubly ionized cells, when the dielectric constant was replaced by the lower bound experimental value of 15 .

\section{RESULTS}

\section{A. Atomic and electronic structure}

The structural parameters of crystalline $\mathrm{SnO}$, as calculated using different exchange-correlation functionals, are summarized in Table I. A clear improvement in the agreement of theoretically predicted and experimentally measured structural parameters can be noted, when moving from GGA to a hybrid-functional level of theory. The intralayer distances are captured perfectly by both the HSE06 and the PBE0 functionals. In contrast, the inter-layer distances, where vdWs interactions dominate, show little improvement and remain $\sim 3 \%$ larger than the experimental values. The DFT-D2 method, that includes approximate corrections for the dispersion forces, resulted in inter-layer distances closer to the experimental values than the hybrid functionals, but was found to overestimate the intralayer distances.

As is typical of semilocal functionals, PBE is seen to underestimate the electronic band gap of $\mathrm{SnO}$ by $35 \%$ when compared to values measured experimentally. Despite their agreement on the structural parameters, the two hybrid functionals result in very different electronic gaps. While HSE06 improves upon the PBE values, giving excellent agreement with the experimental results, the PBE0 hybrid functional overestimates both the indirect $(\Gamma \rightarrow M)$ and the direct $(\Gamma \rightarrow$ $\Gamma)$ gaps by over $0.6 \mathrm{eV}$. Even more surprisingly while providing great improvement in the lattice parameters, the vdWs functional produces an even smaller band gap than that found with PBE. In fact, a semimetallic band structure of $\mathrm{SnO}$ is predicted with a band gap of merely $0.025 \mathrm{eV}$.

Capturing the correct electronic structure is crucial for formation energy calculations of defects. In many ionic (and nonionic) materials, including $\mathrm{SnO}_{2}$ [23], the size of the band gap is seen to be highly sensitive to lattice vector changes. To estimate the magnitude of the error, that may arise from the $3 \%$ change in the interlayer distances calculated by HSE06, we investigated the dependence of the fundamental (indirect) band-gap of $\mathrm{SnO}$ on the lattice constants of the

TABLE I. Lattice parameters, formation energies and electronic band gaps of $\mathrm{SnO}$ calculated with different exchange-correlation functionals.

\begin{tabular}{lccrrc}
\hline \hline & Exp. & PBE & HSE06 & PBE0 & vdW-D2 \\
\hline$a(\AA)$ & $3.8011^{\mathrm{a}}$ & 3.867 & 3.801 & 3.800 & 3.842 \\
$c(\AA)$ & $4.8351^{\mathrm{a}}$ & 5.042 & 4.984 & 4.984 & 4.820 \\
$u$ & $0.2381^{\mathrm{a}}$ & 0.231 & 0.231 & 0.231 & 0.242 \\
$\mathrm{E}^{F}(\mathrm{eV})$ & $-2.96^{\mathrm{b}}$ & -2.59 & -2.505 & -2.517 & - \\
$\mathrm{E}_{G}^{\text {ind }}(\mathrm{eV})$ & $0.7^{\mathrm{c}}$ & 0.45 & 0.677 & 1.305 & 0.025 \\
$\mathrm{E}_{G}^{\text {dir }}(\mathrm{eV})$ & $2.6^{\mathrm{c}}$ & 1.93 & 2.677 & 3.325 & 2.123 \\
\hline \hline
\end{tabular}

\footnotetext{
${ }^{a}$ Reference [6].

${ }^{\mathrm{b}}$ Reference [42].

${ }^{\mathrm{c}}$ Reference [19].
}

litharge structure. The results are summarized in the center panel of Fig. 1. We observe that for any fixed value of the intralayer distance the fundamental band gap of $\mathrm{SnO}$ is almost independent of the interlayer distance. In fact, at the ideal value of $a=3.801 \AA$ determined by HSE06, the band gap lies in the range of $0.67-0.70 \mathrm{eV}$ throughout a variation of $c$ by $\pm 2 \%$ in excellent agreement with the experimental value of $0.7 \mathrm{eV}$. Furthermore, a difference of less than $10 \mathrm{meV}$ was found between the indirect band gaps obtained using the experimental lattice and the HSE06 relaxed lattice.

According to Fig. 1, the overestimation of interlayer distances by the HSE06 functional would introduce negligible errors to the band gap and, consequently, to the defect levels inside the gap. Despite this, inconsistencies in the structural parameters could still result in seizable errors. Local atomic relaxations following the introduction of a dopant element, for example, could be affected by the proximity of the $\mathrm{SnO}$ layers. In particular, where interstitial atoms are concerned strong interlayer distance dependence could be expected. Such concerns will be addressed in more detail in Sec. III E.

\section{B. Analysis of $p$-type dopants}

The PBE pre-screening results for $p$-type dopants in tin monoxide are summarized in Fig. 2. Geometric shapes identify the stable substitutional site of each element, while the color of the geometric shape shows the ionization state of the defect on this site. Here, the Fermi level is fixed at the valence band maximum. Acceptor doping is achieved if the impurity atom is negatively charged under these conditions (identified by a blue color in Fig. 2). Although no elements were found to act as acceptors for Fermi levels at the valence band maximum (i.e., degenerate $p$-type conductivity could not be achieved), six defect species showing promising shallow acceptor behavior were discovered. These impurities- Li, Na, K, Rb, $\mathrm{Cs}$, and Ag-highlighted by a dashed outline in Fig. 2 were found to result in an unoccupied perturbed host state, with no localized electronic defect states within the fundamental band gap. Similar to the activation energy of $96 \mathrm{meV}$ reported experimentally for the tin vacancy [4], the ionization levels for these proposed acceptors evaluated with the PBE level of theory were estimated to lie within $100 \mathrm{meV}$ above the VBM.

In addition to the elements above, the charge states of the less favorable substitutions (indicated in Fig. 2 via small colored circles), revealed that $\mathrm{Os}_{\mathrm{O}}$ and $\mathrm{In}_{\mathrm{O}}$ should also result in hole generation. Unfortunately, for both of these elements, even under Sn-rich growth conditions, O-site substitution is found to be extremely unfavorable when compared to Sn-site substitution. An extra $1.7 \mathrm{eV}$ is required to form $\mathrm{Os}_{\mathrm{O}}$ and an extra $4.2 \mathrm{eV}$ to form $\mathrm{I}_{\mathrm{O}}$. We can hypothesize, that apart from sophisticated chemical growth techniques, that allow for targeted site substitution, $p$-type doping with these two elements would be exceedingly challenging to achieve in practice. In particular, if reactive methodologies that are important for large scale thin-film deposition, such as sputtering, were used.

We note that the majority of the elements seem to favor the substitutional $\mathrm{Sn}$ site, $\mathrm{D}_{\mathrm{Sn}}$, marked by triangles in Fig. 2. However, for some atoms, shaded in light-gray in Fig. 2, Snsite substitution could not be successfully stabilized. For this subset of elements strong local atomic relaxations, following 


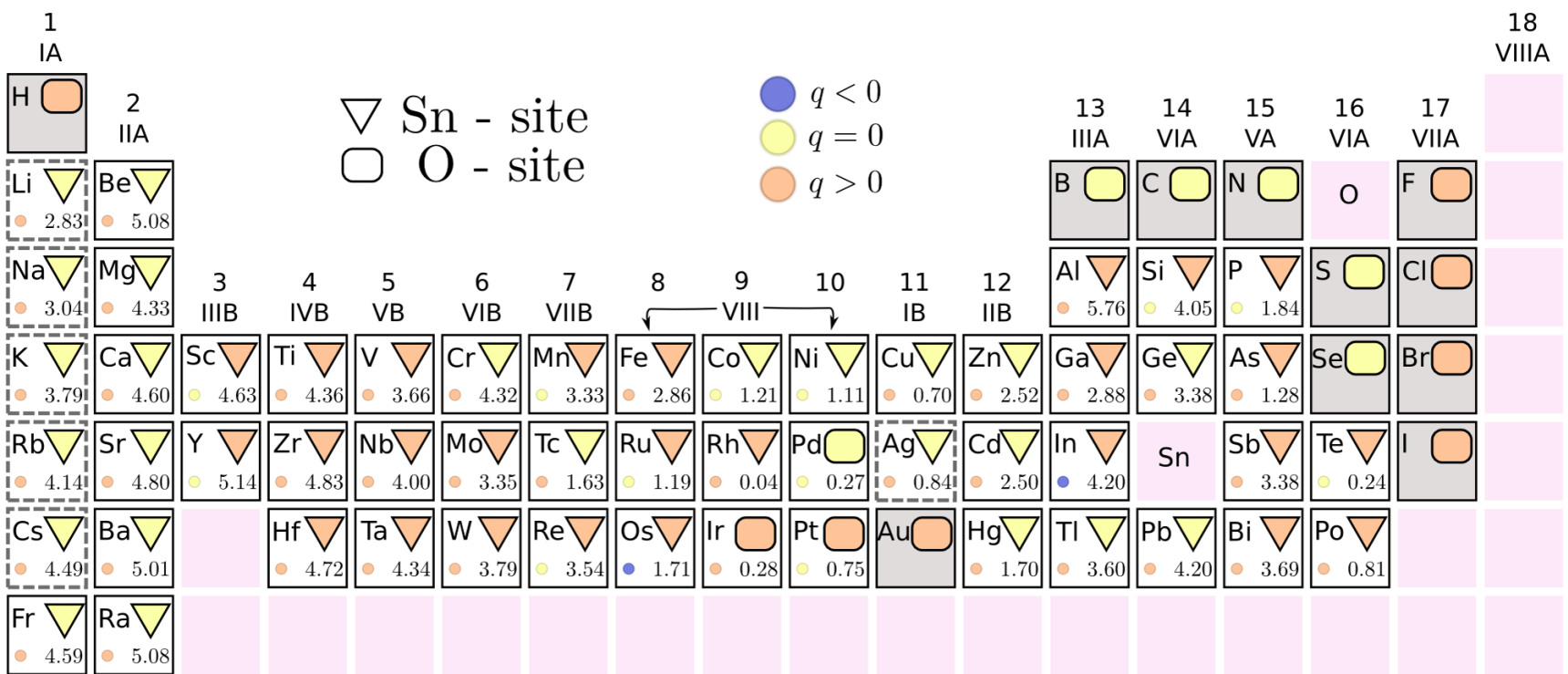

FIG. 2. PBE prescreening results for $p$-type dopants in SnO. Geometric shapes indicate the energetically favored substitution of the two sites available. $D_{S n}$ site is marked by a triangle, $D_{O}$ by a rectangle. The color of the shape indicates the charge state (stable when the Fermi level is at the VBM): rose-positive, yellow-neutral, blue-negative. For each element, the energy difference (in eV) between the two substitutional sites is indicated in the bottom right corner. The stable charge state of the alternative site is indicated by the small colored circle. Elements with thermodynamic transition levels to an acceptor state close to the VBM are indicated by a dashed outline. Elements with no stable $\mathrm{D}_{\mathrm{Sn}}$ substitution are shaded in gray.

the introduction of the defect, lead to structural environments that should be more aptly regarded as defect complexes.

The lightest of the elements-hydrogen — when substituted for tin, moves out of the central four oxygen coordinated position (shown in Fig. 1) and forms instead a single $\mathrm{O}-\mathrm{H}$ bond with one of the neighboring oxygen atoms. The calculated bond length at the stable charge state was found to be $0.98 \AA$, comparable to the bond length of $0.97 \AA$ in an $\mathrm{H}_{2} \mathrm{O}$ molecule (obtained from a reference phase calculation). The resulting defect, therefore, resembles more closely a hydrogen interstitial neighboring a tin vacancy, than a $\mathrm{H}_{\mathrm{Sn}}$.

Carbon, introduced onto a tin site, rejects the tetrahedral oxygen coordination in favor of a $\mathrm{CO}_{2}$ molecule formation, but unlike hydrogen remains in the central position. Two oxygens are acquired from the neighboring tins by breaking the corresponding $\mathrm{Sn}-\mathrm{O}$ bonds, while the other two oxygens are pushed away from the defect site. The $\mathrm{C}-\mathrm{O}$ bond length calculated in this configuration is $1.17 \AA$, akin to the distance in an isolated $\mathrm{CO}_{2}$ molecule of $1.16 \AA$ [43].

Boron is seen to adopt a mixture of the two atomic structures formed by carbon and hydrogen. The element migrates towards one of the neighboring oxygens, forming a bond at a distance of $1.43 \AA$ A. Simultaneously, two additional oxygens are pulled away from their respective tins, following the boron to form a borate $\left(\mathrm{BO}_{3}\right)$ type environment. These additional oxygen atoms form slightly shorter $\mathrm{B}-\mathrm{O}$ bonds at a distance of $1.38 \AA$. For comparison, bond distances of $1.38 \AA$ are reported for an isolated $\mathrm{BO}_{3}$ molecule and $1.47 \AA$ for an isolated $\mathrm{BO}_{4}[44]$ molecule.

Relaxed local atomic structures of all three defect clusters described above can be found in Ref. [24]. Intriguingly, the formation of these heavily altered local environments is still found to be energetically favored over the simple O-site substitution-for hydrogen by $0.01 \mathrm{eV}$, carbon by $0.18 \mathrm{eV}$ and boron by an astonishing $5.08 \mathrm{eV}$. In all three cases, the resulting defect clusters are found to be stable in a $q=+1$ charge state at the VBM, that would counter rather than enhance $p$-type conductivity.

The remaining atoms that fail to form Sn-site substitutions (halides, chalcogens, nitrogen, and gold) are expelled from the substitutional site into the interstitial space. A representative structure of the resulting geometries is included in Ref. [24]. These configurations are more appropriately described as $\left(D_{\text {pir }}^{\mathrm{i}}+\mathrm{V}_{\mathrm{Sn}}\right)$ defect clusters. In all cases, the resulting atomic arrangements were found to be stable in a negative charge state, indicating that such defect clusters could enhance $p$ type conductivity. Moreover, formation energies competitive with simple O-site substitution were observed for iodine, bromine, chlorine, and gold. A detailed analysis of the cause for the energetic favoring of these clusters is outside the scope of the current study, and will be addressed in a following publication. In this work, we focus on substitutional doping.

The acceptor character of the six substitutional elements identified in Fig. 2 was verified using hybrid functional level of theory. Formation energies as a function of the Fermi level calculated using the HSE06 functional are shown in the right panel of Fig. 3. All six elements are observed to retain their shallow acceptor behavior using the higher level of theory. In opposition to the PBE results, the charge neutral state is not found to be stable at the VBM using hybrid calculations, instead a direct $\epsilon(+/-)$ thermodynamic transition is seen. The shallowest transition level is observed to belong to silver at $49 \mathrm{meV}$ and the deepest to cesium at $93 \mathrm{meV}$ above the valence band. Small activation energies for $p$-type conductivity are, hence, expected with these dopant elements.

Formation energies of the most common intrinsic defects in tin monoxide, were also calculated and are shown in the 


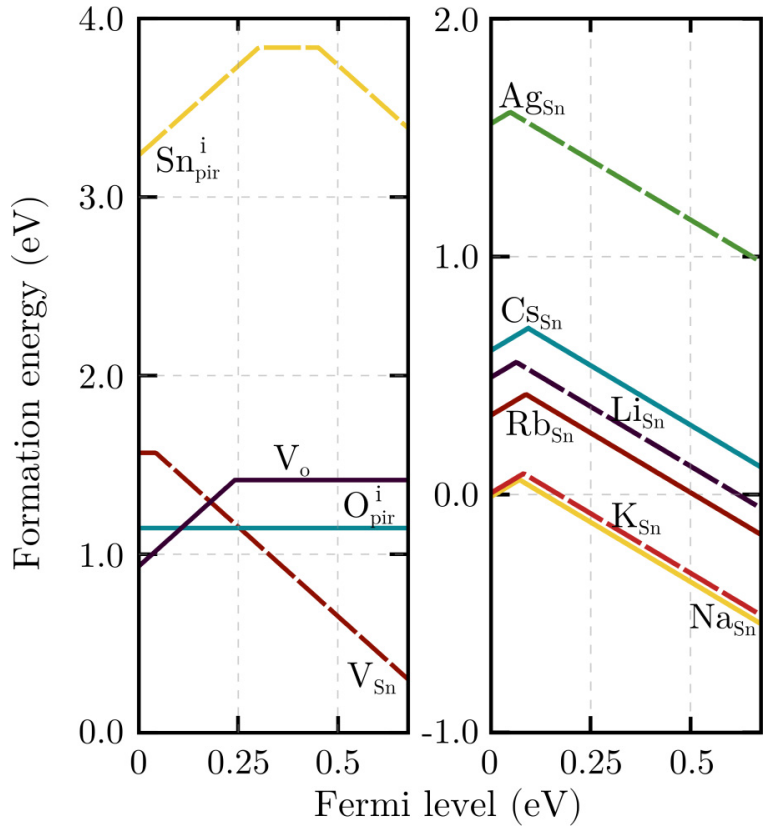

FIG. 3. Defect formation energies as a function of the Fermi level inside the band gap of $\mathrm{SnO}$ (HSE06). Left panel shows intrinsic defects in $\mathrm{SnO}$, right panel shows the preselected $p$-type dopants. Snrich conditions are assumed. Only the stable charge state is shown.

left panel of Fig. 3. The oxygen vacancy $\left(\mathrm{V}_{\mathrm{O}}\right)$ is found to be favorable over $\mathrm{V}_{\mathrm{Sn}}$ formation for Fermi levels near the valence band. In contrast, all alkali impurities are predicted to have formation energies lower than this compensating defect. Our calculated $\epsilon(2+/ 0)$ transition level for $\mathrm{V}_{\mathrm{O}}$ at $0.24 \mathrm{eV}$ is in agreement with the study in Ref. [5]. The work of Allen et al. [6], using a fitted PBE0-vdw type functional, finds the same ionization level to be shifted below the valence band edge. This appears to be inconsistent with experimental observations that report the Fermi level of nominally undoped $\mathrm{SnO}$ to be pinned at $0.1-0.2 \mathrm{eV}$ above the VBM [41].

From the suggested $p$-type impurities, silver is found to require the most energy to incorporate, yet still offers formation energies competitive with the tin vacancy. In agreement with previous studies $[3,5,6]$, shallow defect behavior for $V_{S n}$ is observed. We report the shallowest acceptor ionization level, compared to the two preceding hybrid functional studies of tin monoxide [5,6]. Our estimate for the $\epsilon(0 / 2-)$ transition of just $40 \mathrm{meV}$ above the VBM, is lower than the $\epsilon(0 /-)$ transition at $120 \mathrm{meV}$ predicted by the work of Varley et al. [5], where the HSE functional with an increased $32 \%$ fraction of the exact-exchange was employed. In Ref. [6], an even deeper $(390 \mathrm{meV})$ ionization level was suggested, again appearing to be at odds with the low activation energies $(<100 \mathrm{meV})$ reported experimentally $[4,41]$.

The alkali metals were found to have low formation energies, with exothermic defect formation predicted in $n$-type $\mathrm{SnO}$ for all elements, except cesium. As such, the incorporation of the alkali metals into the host lattice appears to be easily realizable experimentally. To further validate our results, we calculated the expected hole concentrations in thermal equilibrium, for $\mathrm{SnO}$ films containing acceptor impurities together with the native defects shown in the left panel of Fig. 3. These results can be found in Ref. [24]. Our investigations reveal that alkali metals increase the freecharge-carrier densities by orders of magnitude, when compared to intrinsic defects only. Silver, however, was found to offer no significant improvement upon undoped $\mathrm{SnO}$.

\section{Analysis of $\boldsymbol{n}$-type dopants}

The PBE pre-screening results for $n$-type dopants in tin monoxide are summarized in Fig. 4. Analogous to $p$-type results, the geometric shapes identify the stable substitutional site (the Fermi level is fixed at the conduction band minimum), while the color of the shape shows the stable charge state of the defect. The light-gray shading indicates defect elements where $\mathrm{Sn}$-site substitution could not be stabilized as discussed at length in the preceding section.

The formation energy of a charged defect depends on the position of the Fermi level. It follows, that a change in the energy difference between the two substitutional sites, $E_{D_{\mathrm{Sn}}}^{F}\left(q, \Delta \epsilon_{F}\right)-E_{D_{\mathrm{o}}}^{F}\left(q^{\prime}, \Delta \epsilon_{F}\right)$, for elements with $q \neq q^{\prime}$ is seen. As a consequence, the stable substitutional site in $n$-type $\mathrm{SnO}$ may be different from $p$-type $\mathrm{SnO}$. In fact, both $\left(\mathrm{Cl}_{\text {pir }}^{\mathrm{i}}+\right.$ $\left.\mathrm{V}_{\mathrm{Sn}}\right)$ and $\left(\mathrm{Au}_{\mathrm{pir}}^{\mathrm{i}}+\mathrm{V}_{\mathrm{Sn}}\right)$ defect clusters become favorable over $\mathrm{O}$-site substitution, when the Fermi level is at the CBM. In opposition, $\mathrm{C}_{O}$ substitution becomes energetically favorable over the $\mathrm{CO}_{2}$ molecule formation in the $\mathrm{Sn}$ site.

Electrons are successfully donated into the host material if a substitutional element is positively charged, when the Fermi level lies at the conduction band edge (identified by a rose color in Fig. 4). The strict requirement of degenerate $n$-type doping, however, as seen from the figure would result in no suitable donors for $\mathrm{SnO}$. Nevertheless, $n$-type tin monoxide was demonstrated experimentally by Hosono et al. [4] using antimony doping, with activation energies of $92 \mathrm{meV}$. The shallow donor behavior of the $\mathrm{Sb}$ impurities was later verified by the computational work of Varley et al. [5], with an estimated ionization level at $120 \mathrm{meV}$ below the CBM. Given the above information, we conclude that elements with thermodynamic transition levels below the CBM but close to those of antimony could also successfully dope $\mathrm{SnO} n$ type. A total of fifteen defects was identified to fall into this category. The corresponding atoms are marked by a dashed outline in Fig. 4 . Most of the donor impurities selected demonstrate shallow behavior, i.e., the additional electrons are seen to occupy a perturbed host state, with localized electronic defect levels only forming above the conduction band minimum of the host [45].

Just like in the case of acceptor defects, elements that appear to be suitable donors on the less energetically favorable site were found. These include most of the transition metals in groups IV-B and V-B. However, as seen in Fig. 4 elements that show $n$-type behavior when substituting on an oxygen site, also require over $3 \mathrm{eV}$ more energy to form this substitution. Regrettably, such defects would be very difficult to realize in practice and, as a consequence, were excluded from further investigations.

Formation energies of potential $n$-type dopants calculated using hybrid-functional level of theory are summarized in Fig. 5. Elements with lower formation energies are shown in the left panel of the figure, while those with relatively high formation energies are shown on the right hand side. 


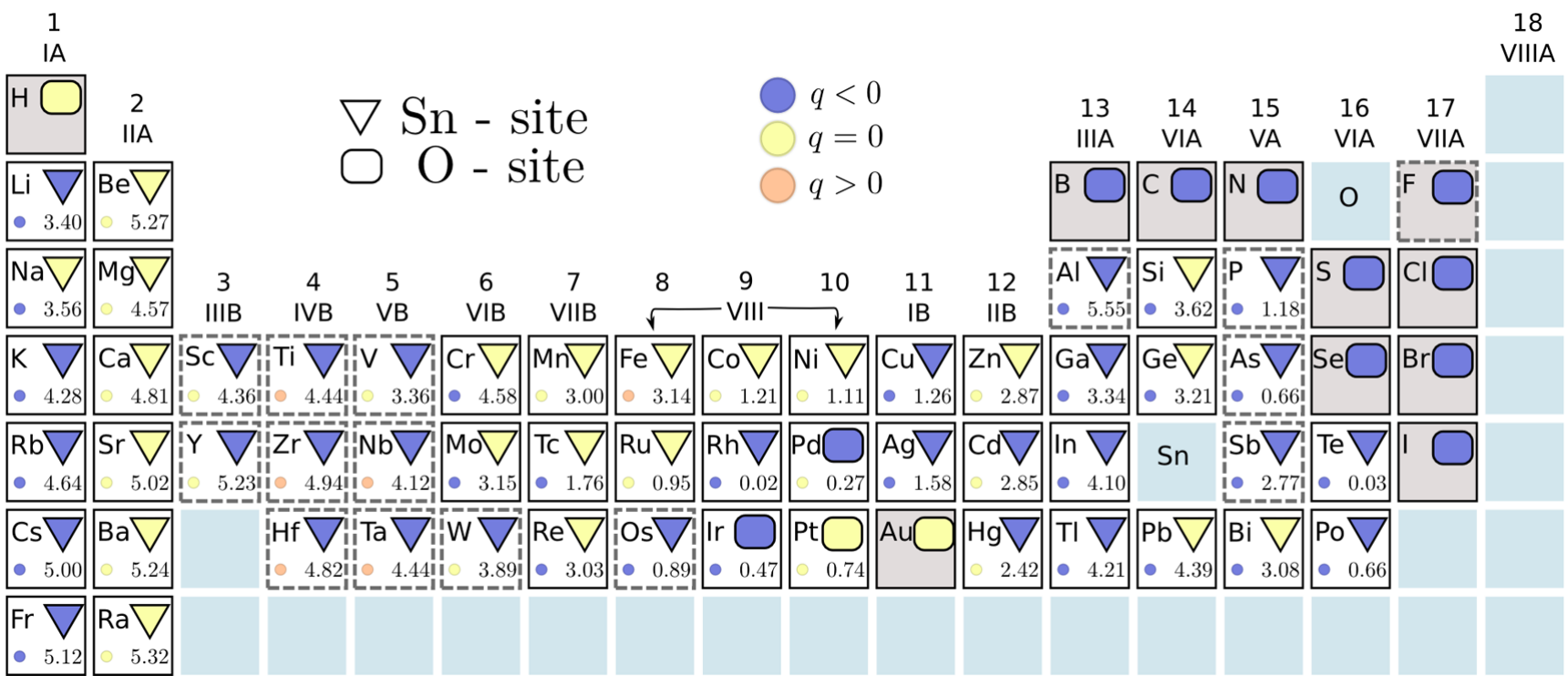

FIG. 4. PBE prescreening results for $n$-type dopants in SnO. Geometric shapes indicate the energetically favored substitution of the two sites available. $D_{\mathrm{Sn}}$ site is marked by a triangle, $\mathrm{D}_{\mathrm{O}}$ by a rectangle. The color of the shape indicates the charge state (stable when the Fermi level is at the CBM): rose - positive, yellow—neutral, blue — negative. For each element, the energy difference (in eV) between the two substitutional sites is indicated in the bottom right corner. The stable charge state of the alternative site is indicated by the small colored circle. Elements with thermodynamic transition levels to a donor state close to the CBM are indicated by a dashed outline. Elements with no stable $\mathrm{D}_{\mathrm{Sn}}$ substitution are shaded in gray.

Substitutional vanadium is the only defect that displays a change of character (shallow to deep donor) between hybrid functional and GGA levels of theory. The energy required to form the neutral charge state of this element is significantly lowered by spin-polarization using HSE06. In contrast, a nonpolarized ground state is found by PBE. All other elements are seen to retain their shallow donor character.

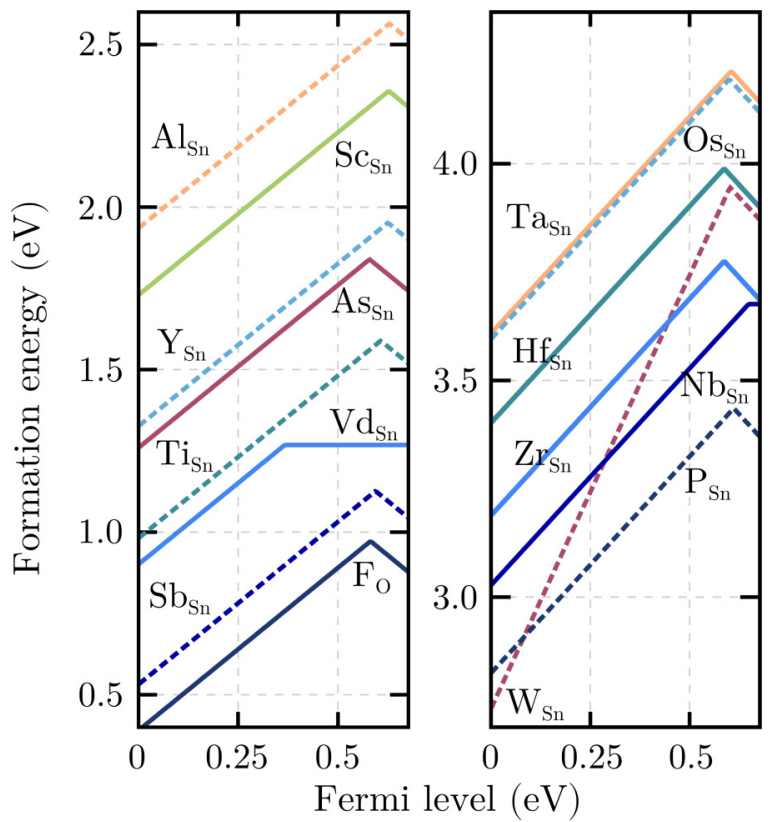

FIG. 5. Defect formation energies as a function of the Fermi level for preselected $n$-type dopants (obtained using the HSE06 xc functional). $\mathrm{Vd}_{\mathrm{Sn}}$ here stands for vanadium on a $\mathrm{Sn}$ site to avoid confusion with a tin vacancy. Sn-rich conditions are assumed. Only the stable charge state is shown.
Most of the identified $n$-type impurities undergo a direct $\epsilon(+/-)$ transition at Fermi levels less than $100 \mathrm{meV}$ below the CBM. $\mathrm{Al}_{\mathrm{Sn}}, \mathrm{Sc}_{\mathrm{Sn}}$, and $\mathrm{Y}_{\mathrm{Sn}}$ exhibit shallowest ionization levels just $\sim 50 \mathrm{meV}$ below the CBM, closely followed by $\mathrm{P}_{\mathrm{Sn}}$, $\mathrm{Ti}_{\mathrm{Sn}}, \mathrm{Ta}_{\mathrm{Sn}}, \mathrm{Os}_{\mathrm{Sn}}$, and $\mathrm{W}_{\mathrm{Sn}}$. All of the aforementioned defects are predicted to have thermodynamic transition levels closer to the conduction band than antimony-the only element previously reported to successfully dope $\mathrm{SnO} n$ type [4]. $\mathrm{Zr}_{\mathrm{Sn}}$, $\mathrm{Hf}_{\mathrm{Sn}}$, and $\mathrm{F}_{\mathrm{O}}$ exhibit thermodynamic transition levels slightly deeper than those of $\mathrm{Sb}_{\mathrm{Sn}}$ by roughly $10 \mathrm{meV}$. All of these defects are deemed likely to act as shallow donors in tin monoxide if incorporated successfully. The exact values of all thermodynamic transition levels are summarized in Table II.

The thermodynamic transition from a donor into an acceptor state close to the conduction band edge suggests that, after

TABLE II. Thermodynamic transition levels for $n$-type dopant elements in $\mathrm{SnO}$ evaluated with hybrid functional theory. Values are shown in $\mathrm{meV}$, as distance away from the conduction band edge. $\mathrm{Vd}_{\mathrm{Sn}}$ here stands for vanadium on a $\mathrm{Sn}$ site to avoid confusion with a tin vacancy.

\begin{tabular}{lccc}
\hline \hline Dopant element & $(+/-)$ & Dopant element & $(+/-)$ \\
\hline $\mathrm{Al}_{\mathrm{Sn}}$ & 47 & $\mathrm{Os}_{\mathrm{Sn}}$ & 77 \\
$\mathrm{Sc}_{\mathrm{Sn}}$ & 52 & $\mathrm{Sb}_{\mathrm{Sn}}$ & 83 \\
$\mathrm{Y}_{\mathrm{Sn}}$ & 52 & $\mathrm{Zr}_{\mathrm{Sn}}$ & 90 \\
$\mathrm{P}_{\mathrm{Sn}}$ & 66 & $\mathrm{Hf}_{\mathrm{Sn}}$ & 90 \\
$\mathrm{Ti}_{\mathrm{Sn}}$ & 70 & $\mathrm{~F}_{\mathrm{O}}$ & 95 \\
$\mathrm{Ta}_{\mathrm{Sn}}$ & 74 & $\mathrm{As}_{\mathrm{Sn}}$ & 96 \\
\hline Dopant element & $(+/ 0)$ & $(0 /-)$ & $(2+/-)$ \\
\hline $\mathrm{Vd}_{\mathrm{Sn}}$ & 311 & -144 & $\mathrm{~N} / \mathrm{A}$ \\
$\mathrm{Nb}_{\mathrm{Sn}}$ & 30 & -130 & $\mathrm{~N} / \mathrm{A}$ \\
$\mathrm{W}_{\mathrm{Sn}}$ & $\mathrm{N} / \mathrm{A}$ & $\mathrm{N} / \mathrm{A}$ & 75 \\
\hline \hline
\end{tabular}


a certain dopant concentration is exceeded, the Fermi level of the system will be pinned below the CBM. $\mathrm{Nb}_{\mathrm{Sn}}$ is, notably, the only shallow donor with a stable neutral charge state: the $\epsilon(+/ 0)$ transition is calculated to lie only $30 \mathrm{meV}$ below the conduction band and self-compensation is anticipated at $130 \mathrm{meV}$ above the CBM. The shallow acceptor transition and the delayed onset of self-compensation suggest niobium as a very promising $n$-type dopant.

So far, donor formation has only been considered in isolation, however in a realistic material different types of defects coexist. While compensation via extrinsic defects may be avoided with careful control of the experimental conditions, native defect formation is much more challenging to suppress. As seen from the left panel of Fig. 3, oxygen related defects do not hinder the creation of $n$-type carriers, but the low formation energy of $\mathrm{V}_{\mathrm{Sn}}$ suggests strong compensation of the donated electrons, especially in $n$-type $\mathrm{SnO}$.

Our estimated defect concentrations (see Fig. S3 in Ref. [24]) reveal that $\mathrm{Sb}_{\mathrm{Sn}}$ and $\mathrm{F}_{\mathrm{O}}$ are the only two donor elements that are dominant over $\mathrm{V}_{\mathrm{Sn}}$ in thermal equilibrium. The lower formation energy of $\mathrm{F}_{\mathrm{O}}$, when compared to $\mathrm{Sb}_{\mathrm{Sn}}$, suggests the former to be a more effective donor. Furthermore, oxygen-poor conditions that favor $\mathrm{F}_{\mathrm{O}}$ creation, are most appropriate for minimizing the concentration of compensating $\mathrm{V}_{\mathrm{Sn}}$ defects. Unfortunately, low defect concentrations are predicted for both donors in thermal equilibrium.

Off-equilibrium defect concentrations of impurities would therefore need to be incorporated into $\mathrm{SnO}$ for $n$-type conductivity to be achieved. If formed, $\mathrm{D}_{\mathrm{Sn}}$ defects would have to overcome an energy barrier to be able to dissociate and diffuse out of the film. The dissociation barrier for $\mathrm{D}_{\mathrm{Sn}} \rightarrow \mathrm{V}_{\mathrm{Sn}}+\mathrm{D}^{\mathrm{i}}$ is the sum between the defect binding energy and the migration barrier of $\mathrm{D}^{\mathrm{i}}$. If the formation energy on an interstitial site is significantly higher than the substitutional site, the defect will not dissociate. As an example, we use the shallowest predicted donor-niobium. The calculated formation energy of $\mathrm{Nb}_{\text {pir }}^{\mathrm{i}}$ in $n$-type $\mathrm{SnO}$ with PBE level of theory is $4.032 \mathrm{eV}$. In an $\mathrm{O}$-site substitution, $\mathrm{Nb}_{\mathrm{O}}$, is even more unfavorable at $7.245 \mathrm{eV}$. On the same level of theory, $\mathrm{V}_{\mathrm{Sn}}$ formation requires $0.873 \mathrm{eV}$ of energy and $\mathrm{Nb}_{\mathrm{Sn}}-3.059 \mathrm{eV}$. A dissociation barrier of at least $1.85 \mathrm{eV}$ is therefore expected, preventing $\mathrm{Nb}_{\text {Sn }}$ from diffusing out of the material at room temperature. Other interstitial atoms are discussed in detail in Sec. III E.

\section{Dopants and transparency}

Ample experimental studies of nano- and polycrystalline tin monoxide report transparency of $70 \%$ or greater in the visible range [14]. Optical properties can also be enhanced further via applied stress/strain or through external doping [46-48]. As such, the preservation of transparency may also be a relevant criteria when selecting dopants for $\mathrm{SnO}$ targeted towards specific applications. In this section, we comment on the defect energy levels, introduced into the optical band gap via external doping, that could have a disruptive impact on the transparency of the material. Such defect levels are illustrated in Fig. 6 for all acceptor and donor impurities found. We note that the folding of the band structure-a by-product of the large supercell size used-introduces energy states below the first conduction band of tin monoxide at $\Gamma$. Such states in regions more than $\sim 2 \mathrm{eV}$ above the valence band maximum (indicated by dark blue in Fig. 6) arise from indirect conduction band edges. A newly incorporated defect state in this energy range is, therefore, challenging to distinguish from the host states. Such distinction was not attempted in this work and we focus our attention only on the region up to $2.1 \mathrm{eV}$ inside the optical band gap.

We observe that six out of the fourteen $n$-type defects (vanadium is concluded not to be an effective dopant) introduce defect states outside of the fundamental band gap, but close to the midregion of the direct optical $(\Gamma \rightarrow \Gamma)$ gap of SnO. A likely ramification of such defect states are color centers, that could negatively effect the transparency. Upon zirconium doping, states inside the fundamental band gap are seen to emerge. The close proximity of the defect states to the valence band edge suggests that they would not play a significant role in optical absorption, if only direct $\Gamma \rightarrow \Gamma$ transitions were considered. Nevertheless, defect states near the valence band edge at $\Gamma$ lie close to the middle of the direct $M \rightarrow M$ gap. In a similar fashion to $\mathrm{Zr}$, tin monoxide doping with $\mathrm{Ta}, \mathrm{Hf}$, and $\mathrm{Nb}$ would trigger defect state formation in the neighborhood of the valence band, closing the transparency window at the $M$ point. Fortunately, four of the $n$-type dopant elements- $\mathrm{F}_{\mathrm{O}}, \mathrm{P}_{\mathrm{Sn}}, \mathrm{Sb}_{\mathrm{Sn}}, \mathrm{As}_{\mathrm{Sn}}$ - are observed to result in a mostly unaltered host band structure. These elements are suitable candidates for synthesizing a transparent $n$-type $\mathrm{SnO}$.

In the case of acceptors, doping with $\mathrm{Ag}_{\mathrm{Sn}}, \mathrm{Li}_{\mathrm{Sn}}$, and $\mathrm{Na}_{\mathrm{Sn}}$ induces defect state formation deep inside the optical band gap at $\Gamma$. Just like in the case of donors, such unoccupied defect states are likely to be detrimental to optical transparency. The three heavier alkali metals are seen, however, to create no defect states inside the optical band gap of $\mathrm{SnO}$ and leave the host band structure unaltered upon incorporation. These three substitutional defects- $\mathrm{K}_{\mathrm{Sn}}, \mathrm{Rb}_{\mathrm{Sn}}$, and $\mathrm{Cs}_{\mathrm{Sn}}$ - are excellent candidates for enhancing the number of free-charge-carriers, while simultaneously maintaining the transparency of $p$-type tin monoxide in the visible range.

\section{E. Effects of vdW and interstitials}

As was outlined in previous sections, the layered structure of tin monoxide is held together by dispersion forces that are not accounted for in standard DFT functionals. In Sec. III A, we demonstrated that the intralayer distances are captured remarkably well by HSE06 (as well as PBE0) but the shortcomings become apparent in the interlayer distancesapproximately $3 \%$ shorter than the experimental values. Despite the correct representation of the band structure by the HSE06 functional (evidenced by Fig. 1), questions pertaining to structural relaxations remain. For a substitutional defect, even with significant reduction in the distances between the layers, strong changes are unlikely to result. The much greater proximity of the atoms within the layer suggests they should account for the majority of local relaxations. On the other hand, for interstitial defects the separation between the layers is anticipated to play a role.

Consequently, the PBE (and HSE06) functionals are speculated to perform poorly in evaluating the energetics of interstitial defects. Unfortunately, as shown in Table I, the vdW-type functional produces a semimetallic $\mathrm{SnO}$ band structure and, thus, does not offer an improved description. Erroneous electronic structure could equally result in inaccurate 

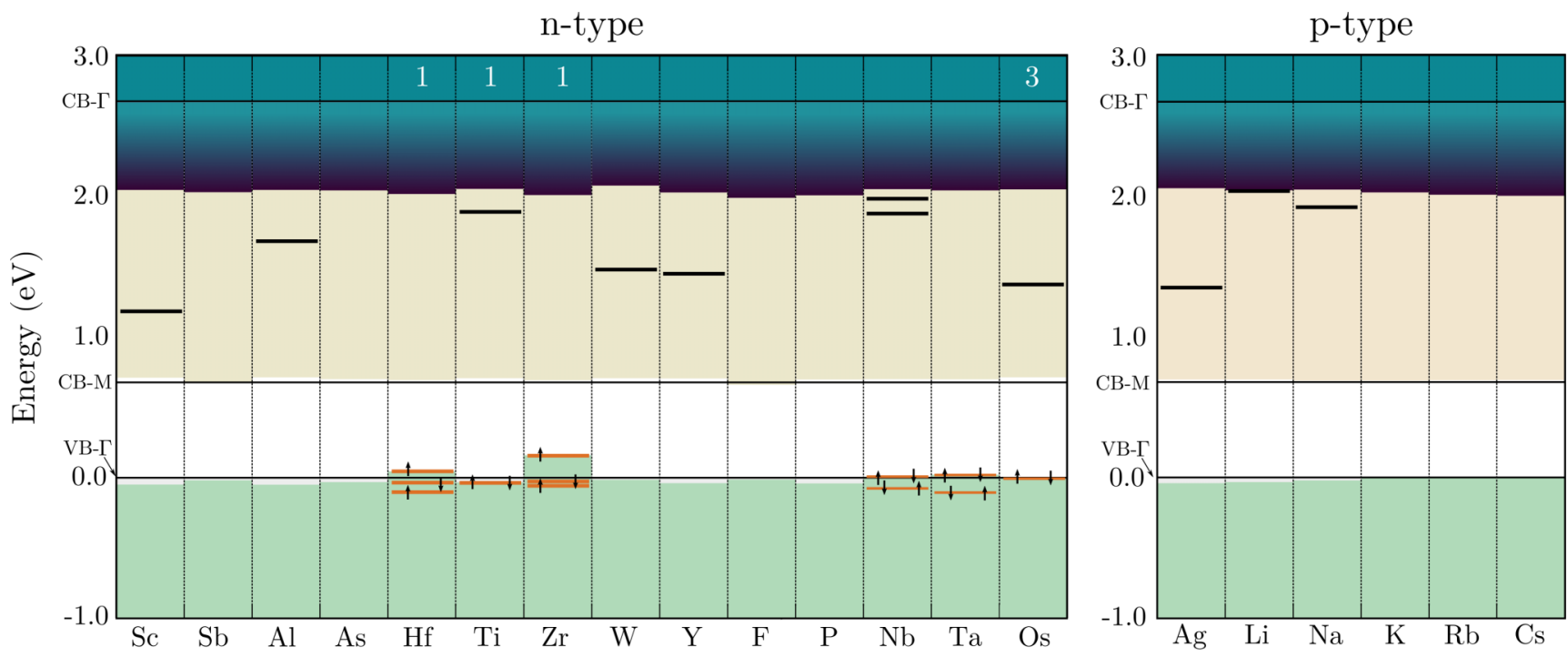

FIG. 6. Midgap electronic defects states of dopant elements in SnO. For donors, a charge state of $q=+1$ is shown, except for tungsten $(q=+2)$. For acceptors, a charge state of $q=-1$ is shown. Black lines indicate the energetic positions of defect states. For defect states close to the valence band, their occupation by spin is shown. Changes in valence and conduction band edges (after band alignment) of the host upon doping are indicated by the edges of the light green and sand colored areas, respectively. Bright blue areas shows the onset of the region below the optical band gap edge in which electronic defect states could not be distinguished. Differences in the spin channel occupation, for structures where spin polarized solutions were found, are indicated by the numbers at the top of the chart.

atomic forces and hence incorrect geometries. The almost nonexistent band gap also rules out the possibility of using such a functional to obtain reliable formation energy information. These issues prevent us from performing a sensible prescreening for interstitial dopant elements in $\mathrm{SnO}$.

Nonetheless, the layered nature of tin monoxide offers plenty of space for interstitial incorporation. These alternative defect sites, could be competitive with the substitutional locations investigated in this work. The energetics of such interstitials are, therefore, significant when considering paths to successful doping of tin monoxide. To support our assertions of having found useful $p$-type and $n$-type dopants we investigated the interstitial positions of those elements using PBE only. The results provide insights on the competition between substitutional and interstitial sites for a given element.

Amongst $n$-type impurities, Os ${ }_{S n}$ was the only element with competitive interstitial incorporation. In a $p$-type $\mathrm{SnO}$, we found the substitutional site to be more stable, however, in $n$-type $\mathrm{SnO}$, the interstitial location is favored by $60 \mathrm{meV}$. The close competition between the substitutional and interstitial sites makes this element unsuitable for doping. All other donors presented were found to be stable on the substitutional site. The lowest relative interstitial energies were calculated for the fluorine atom, requiring an extra $450 \mathrm{meV}$ of energy, and tungsten, requiring an extra $650 \mathrm{meV}$ for interstitial incorporation. The rest of the interstitial defects were found lying over $1 \mathrm{eV}$ higher in energy than the corresponding substitutional sites. The elements for which interstitial incorporation was found to be extremely unlikely were $\mathrm{Al}$ (3.75 eV more energy), $\mathrm{Y}(3.28 \mathrm{eV})$, and $\mathrm{Sc}(2.26 \mathrm{eV})$. In view of these results, it is reasonable to conclude that even if large discrepancies in the calculated formation energies arose from the PBE functional, the $n$-type dopants would still favor substitutional sites.
Most of the donor impurities exhibit amphoteric behavior when incorporated on interstitial sites, i.e., act as compensating defects to the majority free carriers in both an $n$-type and a $p$-type doped material. However, some exceptions are present. On the PBE level of theory we observe $\mathrm{Sb}_{\text {pir }}^{\mathrm{i}}, \mathrm{C}_{\text {pir }}^{\mathrm{i}}, \mathrm{P}_{\text {pir }}^{\mathrm{i}}, \mathrm{F}_{\text {tet }}^{\mathrm{i}}$, and $\mathrm{Y}_{\mathrm{pir}}^{\mathrm{i}}$ to show shallow acceptor behavior. This may offer a potential explanation for the experimental reports of $p$-type properties in $\mathrm{SnO}$ enhanced by both $\mathrm{Sb}$ and $\mathrm{Y}$ doping [21]. However, one needs to keep in mind that the interstitial incorporation is predicted to be extremely unfavorable $>3 \mathrm{eV}$ for $\mathrm{Y}_{\mathrm{pir}}^{\mathrm{i}}$ and $\sim 2 \mathrm{eV}$ for $\mathrm{Sb}_{\mathrm{pir}}^{\mathrm{i}}$.

Silver was the only acceptor found to be more stable in the interstitial site by $550 \mathrm{meV}$, and the only acceptor found to favor the tetrahedral rather than the piramidal interstitial site. The $\mathrm{Ag}_{\text {tet }}^{\mathrm{i}}$ was predicted to act as a shallow donor by PBE, countering the desired $p$-type conductivity. The preference of silver to form interstitials, as well as its hefty market value, makes this element unlikely to be a useful dopant. Substitution on a Sn site was seen to be the most energetically favored position for all alkali metals. The smallest energy difference of $\sim 1 \mathrm{eV}$ was found between the $\mathrm{Li}_{\mathrm{Sn}}$ and $\mathrm{Li}_{\text {pir }}^{\mathrm{i}}$. This energy divide was observed to increase with the size of the alkali metal, with cesium interstitial found to be over $3 \mathrm{eV}$ more expensive to create than the substitution. The alkali metals incorporated on the interstitial sites were seen to act as electron donors with $\epsilon(0 /+)$ transition levels $100 \mathrm{meV}$ below the conduction band. This suggests that dopant elements incorporated on the wrong site would act as compensating centers and should be mitigated.

\section{CONCLUSIONS}

In this work, a thorough search of useful dopants in $\mathrm{SnO}$, aimed at improving the conductivity of the material, was 
presented. The affinity of an element for donating free-carriers when incorporated as a substitutional defect was assessed for every atom in the periodic table. The stability of the substitutional defects, established as useful, was compared with alternative defect locations, eliminating the elements that are not likely to be stable dopants. In this way, five alkali metals- $\mathrm{Li}, \mathrm{Na}, \mathrm{K}, \mathrm{Rb}$, and $\mathrm{Cs}$-were identified as prospective $p$-type carrier generators. Furthermore, thirteen elemnets acting as shallow donors-eight transition metals: $\mathrm{Sc}, \mathrm{Y}, \mathrm{Ti}, \mathrm{Zr}, \mathrm{Hf}, \mathrm{Nb}, \mathrm{Ta}, \mathrm{W}$ as well as As, Al, P, Sb, and F (the only O-site dopant) - were found suitable for enhancing the free-charge-carrier concentrations in $n$-type $\mathrm{SnO}$.

After a close inspection of the optical properties of doped tin monoxide, we further verified that both $n$ - and $p$-type conductivities could be enhanced without detrimental repercussions to transparency. Three of the heavier alkali acceptor impurities $(\mathrm{K}, \mathrm{Rb}, \mathrm{Cs})$ were observed to result in free-carrier generation without altering the bulk band structure. The same conclusions were drawn for $\mathrm{P}, \mathrm{Sb}, \mathrm{As}$, and $\mathrm{F}$ electron-doped $\mathrm{SnO}$ films. Our considerations of interstitial incorporation point towards interstitial elements commonly acting to counter/compensate the carriers donated by the substitutional defects of the same species. Consequently, such offsite defect incorporation should be strictly avoided if doping is to be successfully achieved. Defects with most unfavorable interstitial incorporation were identified as the heaviest alkali metals (for $p$-type dopants) and $\mathrm{Al}, \mathrm{Y}$, and Sc (for $n$-type dopants).

Our methodology shows results in good agreement with previous studies of the native defects in $\mathrm{SnO}$ using the same level of theory and is in line with the experimental findings on both $p$-type and $n$-type SnO. The large variety of potential dopant elements (not yet experimentally explored) discovered through this study, demonstrates tin monoxides' potential for high technological relevance. The strong agreement, between our results and previous studies, hints that many of the doped $\mathrm{SnO}$ variants suggested could be physically synthesized. In this context, we call for further experimental studies to verify our findings.

\section{ACKNOWLEDGMENTS}

Authors would like to thank Fabien Bruneval for useful insights on shallow defect formation. M.G. acknowledges funding from the Swiss National Science Foundation under the Disco Project (No. CRSII2_154474). M.G. and S.G. acknowledges computational resources under the project s707 and J.A.F.-L. acknowledges under the project s752, both from the Swiss National Supercomputing Center (CSCS) in Lugano. This research was partially supported by the NCCR MARVEL, funded by the Swiss National Science Foundation.
[1] X. Pan and L. Fu, J. Electroceram. 7, 35 (2001).

[2] Y. Ogo, H. Hiramatsu, K. Nomura, H. Yanagi, T. Kamiya, M. Hirano, and H. Hosono, Appl. Phys. Lett. 93, 032113 (2008).

[3] A. Togo, F. Oba, I. Tanaka, and K. Tatsumi, Phys. Rev. B 74, 195128 (2006).

[4] H. Hosono, Y. Ogo, H. Yanagi, and T. Kamiya, Electrochem. Solid-State Lett. 14, H13 (2011).

[5] J. Varley, A. Schleife, A. Janotti, and C. Van de Walle, Appl. Phys. Lett. 103, 082118 (2013).

[6] J. P. Allen, D. O. Scanlon, L. F. Piper, and G. W. Watson, J. Mater. Chem. C 1, 8194 (2013).

[7] E. Fortunato, R. Barros, P. Barquinha, V. Figueiredo, S.-H. K. Park, C.-S. Hwang, and R. Martins, Appl. Phys. Lett. 97, 052105 (2010).

[8] H. Yabuta, N. Kaji, R. Hayashi, H. Kumomi, K. Nomura, T. Kamiya, M. Hirano, and H. Hosono, Appl. Phys. Lett. 97, 072111 (2010).

[9] S. A. Miller, P. Gorai, U. Aydemir, T. O. Mason, V. Stevanović, E. S. Toberer, and G. J. Snyder, J. Mater. Chem. C 5, 8854 (2017).

[10] J. A. Caraveo-Frescas, P. K. Nayak, H. A. Al-Jawhari, D. B. Granato, U. Schwingenschlögl, and H. N. Alshareef, ACS nano 7, 5160 (2013).

[11] S. H. Kim, I.-H. Baek, D. H. Kim, J. J. Pyeon, T.-M. Chung, S.-H. Baek, J.-S. Kim, J. H. Han, and S. K. Kim, J. Mater. Chem. C 5, 3139 (2017).

[12] T. Daeneke, P. Atkin, R. Orrell-Trigg, A. Zavabeti, T. Ahmed, S. Walia, M. Liu, Y. Tachibana, M. Javaid, A. D. Greentree, S. P. Russo, R. B. Kaner, and K. Kalantar-Zadeh, ACS nano 11, 10974 (2017).

[13] J. F. Wager, B. Yeh, R. L. Hoffman, and D. A. Keszler, Curr. Opin. Solid State Mater. Sci. 18, 53 (2014).
[14] Z. Wang, P. K. Nayak, J. A. Caraveo-Frescas, and H. N. Alshareef, Adv. Mater. 28, 3831 (2016).

[15] E. Fortunato, P. Barquinha, and R. Martins, Adv. Mater. 24, 2945 (2012).

[16] T. Arai, S. Iimura, J. Kim, Y. Toda, S. Ueda, and H. Hosono, J. Am. Chem. Soc. 139, 17175 (2017).

[17] T. Yang, X. Qin, H.-h. Wang, Q. Jia, R. Yu, B. Wang, J. Wang, K. Ibrahim, X. Jiang, and Q. He, Thin Solid Films 518, 5542 (2010).

[18] F. Zhuge, L. Zhu, Z. Ye, D. Ma, J. Lu, J. Huang, F. Wang, Z. Ji, and S. Zhang, Appl. Phys. Lett. 87, 092103 (2005).

[19] N. Quackenbush, J. Allen, D. Scanlon, S. Sallis, J. Hewlett, A Nandur, B. Chen, K. Smith, C. Weiland, D. Fischer, J. Woicik, B. White, G. W. Watson, and L. Piper, Chem. Mater. 25, 3114 (2013).

[20] V.-A. Ha, D. Waroquiers, G.-M. Rignanese, and G. Hautier, Appl. Phys. Lett. 108, 201902 (2016).

[21] W. Guo, L. Fu, Y. Zhang, K. Zhang, L. Liang, Z. Liu, H. Cao, and X. Pan, Appl. Phys. Lett. 96, 042113 (2010).

[22] F. Oba and Y. Kumagai, Appl. Phys. Express 11, 060101 (2018).

[23] M. Graužinyte, S. Goedecker, and J. A. Flores-Livas, Chem. Mater. 29, 10095 (2017).

[24] See Supplemental Material at http://link.aps.org/supplemental/ 10.1103/PhysRevMaterials.2.104604 for details on chemical potential reference phases, HSE06 relaxation effects, equilibrium defect concentrations and atomic structures corresponding to unstable Sn-site substitutionals.

[25] M. S. Moreno, A. Varela, and L. C. Otero-Díaz, Phys. Rev. B 56, 5186 (1997).

[26] S. Lany and A. Zunger, Phys. Rev. Lett. 98, 045501 (2007).

[27] P. E. Blöchl, Phys. Rev. B 50, 17953 (1994). 
[28] G. Kresse and D. Joubert, Phys. Rev. B 59, 1758 (1999).

[29] G. Kresse and J. Furthmüller, Phys. Rev. B 54, 11169 (1996).

[30] G. Kresse and J. Furthmüller, Comput. Mater. Sci. 6, 15 (1996).

[31] F. Birch, Phys. Rev. 71, 809 (1947).

[32] J. P. Perdew, K. Burke, and M. Ernzerhof, Phys. Rev. Lett. 77, 3865 (1996).

[33] C. Adamo and V. Barone, J. Chem. Phys. 110, 6158 (1999).

[34] J. Paier, M. Marsman, K. Hummer, G. Kresse, I. C. Gerber, and J. G. Ángyán, J. Chem. Phys. 124, 154709 (2006).

[35] J. Heyd, G. E. Scuseria, and M. Ernzerhof, J. Chem. Phys. 118, 8207 (2003).

[36] J. Heyd, G. E. Scuseria, and M. Ernzerhof, J. Chem. Phys. 124, 219906 (2006).

[37] S. Grimme, J. Comp. Chem. 27, 1787 (2006).

[38] Y. Kumagai and F. Oba, Phys. Rev. B 89, 195205 (2014).

[39] D. Broberg, B. Medasani, N. E. R. Zimmermann, G. Yu, A. Canning, M. Haranczyk, M. Asta, and G. Hautier, Comput. Phys. Commun. 226, 165 (2018).
[40] X. Li, L. Liang, H. Cao, R. Qin, H. Zhang, J. Gao, and F. Zhuge, Appl. Phys. Lett. 106, 132102 (2015).

[41] Y. Ogo, H. Hiramatsu, K. Nomura, H. Yanagi, T. Kamiya, M. Kimura, M. Hirano, and H. Hosono, Phys. Status Solidi (a) 206 2187 (2009).

[42] J. G. Stark, H. G. Wallace, and M. McGlashan, Chemistry Data Book (Murray, London, 1982).

[43] G. Glockler, J. Phys. Chem. 62, 1049 (1958).

[44] A. Gupta and J. Tossell, Phys. Chem. Miner. 7, 159 (1981).

[45] S. Lany and A. Zunger, Phys. Rev. B 72, 035215 (2005).

[46] H. Peng, A. Bikowski, A. Zakutayev, and S. Lany, APL Mater. 4, 106103 (2016).

[47] E. Rucavado, M. Graužinytė, J. A. Flores-Livas, Q. Jeangros, F. Landucci, Y. Lee, T. Koida, S. Goedecker, A. Hessler-Wyser, C. Ballif, and M. Morales-Masis, J. Phys. Chem. C 122, 17612 (2018).

[48] J. S. C. Kearney, M. Graužinytè, D. Smith, D. Sneed, C. Childs, J. Hinton, C. Park, J. S. Smith, E. Kim, S. D. S. Fitch, A. L. Hector, C. J. Pickard, J. A. Flores-Livas, and A. Salamat, Angew. Chem. Int. Ed. 57, 11623 (2018). 